\title{
ARTICLE
}

\section{A tangible augmented reality system to support comprehension of radiation shielding}

\author{
Ayako Yano ${ }^{\mathrm{a}^{*}}$, Hiroyuki Ikegami ${ }^{\mathrm{a}}$, Kayo Mouri ${ }^{\mathrm{a}}$, Chisato Mouri ${ }^{\mathrm{b}}$, Shougo Yamashita ${ }^{\mathrm{c}}$ and Hideki Tenzou ${ }^{\mathrm{d}}$ \\ ${ }^{a}$ Advanced Course in Electronics, Information and Communication Engineering, Electronic Systems Course, Kagawa National \\ College of Technology (KNCT), Kouda 551, Takuma-cho, Mitoyo-shi, Kagawa-ken 769-1192, Japan; ${ }^{b}$ Technical Education \\ Support Section, KNCT; ${ }^{c}$ Department of Electronics, KNCT; ${ }^{d}$ Department of Electronic Systems Engineering, KNCT
}

\begin{abstract}
A tangible augmented reality (AR) system was developed to visualize electromagnetic cascades through solid materials. 3-D cascade images calculated by the EGS4 simulation software are overlaid on a USB camera image almost in real time. Learners can observe electromagnetic cascades while handling blocks of real shielding materials. This system was demonstrated at a public science event. By way of a questionnaire survey, it appeared that it was the first time to hear about electromagnetic cascades for about the $90 \%$ of the child participants and that they felt the system made it easy for them to understand how cascades change with differences in both density of shielding materials and incident energies. Some teaching assistants, however, stated that some children had difficulty understanding the incident direction of photons. A new function called time evolution was added to show growing electromagnetic cascades. This system was improved to be suitable and effective tools for primary school educators to promote the radiation awareness by addition of the time-evolution function.
\end{abstract}

\section{Keywords: augmented reality; electromagnetic cascade; radiation shielding; educational tool}

\section{Introduction}

Shielding materials for radiation such as thick concrete are placed around the outside of a nuclear reactor pressure vessel. It's difficult for a lot of people to intuitively understand how radiation shielding works. Cloud chambers are one of the most effective educational tools to observe invisible particles. It's easy to see the tracks generated by charged particles in supersaturated vapor. However, it's difficult to learn about radiation shielding by way of electromagnetic cascades in solid materials. The purpose of this study was to develop an AR system to visualize electromagnetic cascades through solid materials.

\section{AR System}

Figure 1 shows a system overview of the AR system. The learner can observe electromagnetic cascades inside the block via AR technology. This system requires a conventional Windows PC, USB camera, blocks of actual shielding materials, and AR tags. An AR tag is attached to the shielding material block in order to recognize position and orientation of the block from USB camera images. The AR tag-recognition system overlays the electromagnetic cascade image on the USB camera image in real time, at 15 frames per second (fps)

*Corresponding author. Email: a11518@sr.kagawa-nct.ac.jp and $640 \times 480$ pixel resolution. The learner can change the view by manipulating the shielding material block, and can feel the mass of the shielding block and touch the actual materials. The software for this system was developed in Visual C++ 2008 with the ARToolKit library Ver. 2.72 on 64 bit Windows 7. The CPU was a Core i7-2620M (2.70GHz) and the graphic accelerator and the memory were the Mobile Intel(R) HD Graphics and $6 \mathrm{~GB}$, respectively.

\subsection{Data flow diagram}

For preprocessing, the Monte-Carlo simulation is run once on the PC for three different values of energy for each shielding material. And incidents of photons are simulated perpendicularly to the center of each block. The collision data is stored as a text file on the PC. The data consists of positions of collisions, the particle ID and the energy. The parameter of each material is recognized from the AR-tag and the software can retrieve the trajectory information from the disk automatically. 3-D CG line objects are generated based on the data of the cascade calculated in preprocessing. The calculated quantum trajectory is overlaid on the target materials and a dynamic composite image is displayed. The composite image is displayed each time an AR tag is recognized by the software.

\subsection{ARToolKit Library}

ARToolKit Library [1] is a C and C++ language 
software library to aid in developing AR applications. This library enables us to overlay virtual computer graphics images over real-time USB camera images. The ARToolKit video tracking libraries calculate the camera position and orientation relative to physical tags are identified by a matrix bar code written on the tag. The tag can be printed out by a conventional PC printer. The library was used in our system in order to identify the positions of the shielding blocks and draw 3-D line objects of the electromagnetic cascades on the USB camera images of the block, as shown in Figure 1.

\subsection{Electromagnetic cascade Monte-Carlo code EGS4}

A radiation transport of electron, positron, photon and pions can be simulated in arbitrary geometries composed of any element, compound or mixture by EGS4 [2]. EGS4 uses quantum laws of probability in the calculation of electromagnetic cascades. The dynamic range of charged-particle kinetic energies goes from a few tens of keV up to a few thousand $\mathrm{GeV}$. The dynamic range of photon energies lie between $1 \mathrm{keV}$ and several thousand $\mathrm{GeV}$. The geometry of the shielding materials is set as a parallelepiped rectangle and the simulated incident photons are irradiated through the center of the end of the block lengthwise through the block.

\subsection{Comparison of simulation outcome with composite image}

Figure 2-(A) shows the EGS4PICT [3] output of the EGS4 simulation outcome. It shows the electromagnetic cascades calculated for 10 incident photons of $10 \mathrm{MeV}$ inside of a concrete block. Figure 2-(B) shows a composite image in which 3-D line objects representing the electromagnetic cascades are rendered using the same data as in Figure 2-(A). The trajectories generated by pair production, photon scattering and an electron scattering are shown in a circle (a), (b) and (c) in Figure 2 , respectively. These are three features of electromagnetic cascades that the AR system can display using the data calculated by the EGS4.

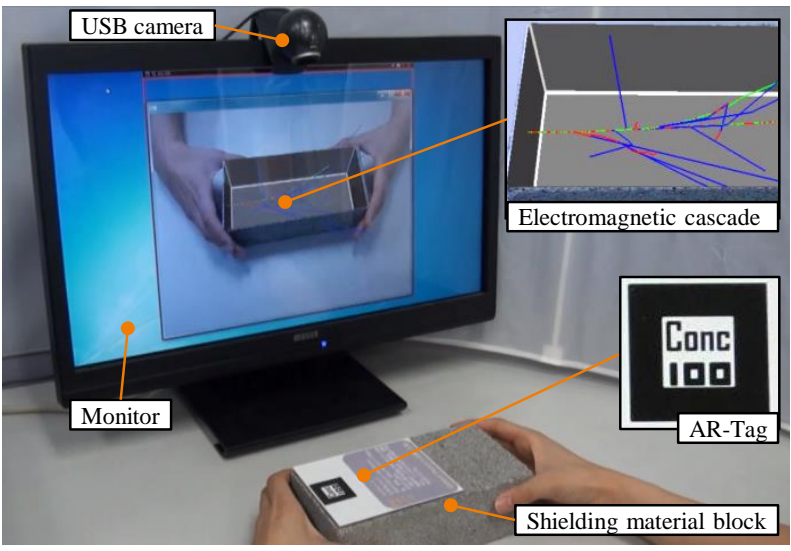

Figure 1. System Overview

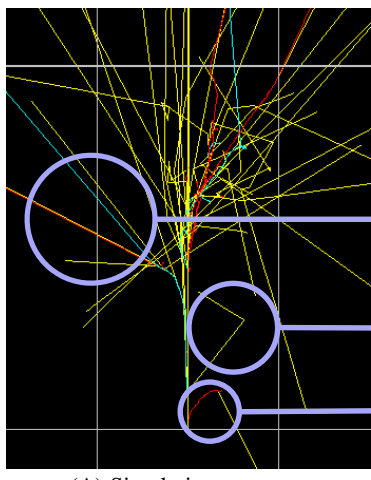

(A) Simulation outcome represented by EGS4PICT

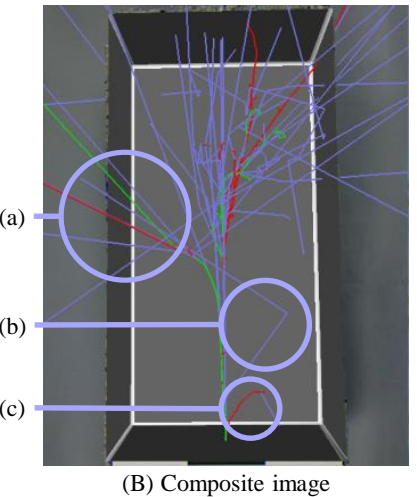

of our AR system
Figure 2. Comparison of simulation outcome with composite image of the AR System

\section{The features of the AR system}

\subsection{The learner can observe cascades while feeling the actual mass}

The simulation software also calculates radiation shielding, but it may be difficult for children to guess the mass of shielding materials from numeric data. The learner can feel the actual mass of shielding materials and more easily understand density. This system shows inside of the shielding block on the display as a dynamic image. Figure 3 is a screen capture with the generated image overlaid on two blocks with different densities. A $10 \mathrm{MeV}$ incident photon is simulated from the left side. In the case of the dense lead block, the cascade contained within the block. The learner can manipulate the shielding block in order to change the view while feeling the force of gravity that the mass of each block exerts.

\subsection{Different shielding materials and incident energies}

Three kinds of materials shown in Table $\mathbf{1}$ are selected as shielding materials for this system. Concrete or lead is often used as shielding materials. A formed polystyrene block which has a density of a few hundred times less than the other materials is added for reference purposes. For the comparison with each other, the three blocks have almost the same surface area for the incident direction of the photon. Due to the density of the lead block, the length of the incident direction is half that of the other blocks so as to make it more manageable while handling. Figure $\mathbf{4}$ is the AR composite image for a $10 \mathrm{MeV}$ photon incident on three different material blocks. The incident photon just passes through in the formed polystyrene block. However, the case of the lead block, the photon and generated radiation are absorbed or stopped in the block. The leaner can see that the development of each cascade in the three different materials is quite different. The line objects of the trajectories for photons, electrons and positrons calculated by EGS4 are showed in different colors: blue, red and green respectively. In order to demonstrate the system at public science events within about 15 minutes, the positions of incident photon is 
fixed at the center of surface of the block and incident energies are set at 1, 10 and $100 \mathrm{MeV}$. Figure 5 shows the electromagnetic cascades by one photon of the three different incident energies on the concrete block. The learner can compare the growth of cascades by changing the AR tag.

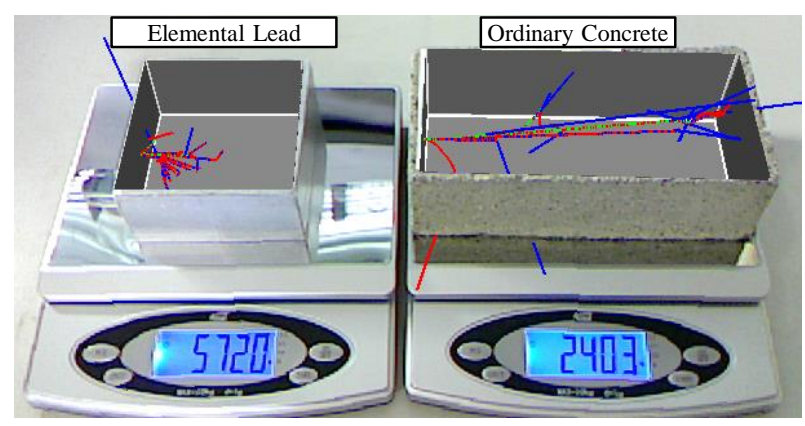

Figure 3. A captured image of two blocks with different density. In the case of the denser lead block, the cascade generated by a $10 \mathrm{MeV}$ photon is stopped within the block.

Table 1. This Parameter of each block

\begin{tabular}{|c|c|c|c|c|c|}
\hline \multirow{2}{*}{ Material } & Density & Weight & \multicolumn{3}{|c|}{ Size of Block [cm] } \\
\cline { 4 - 7 } & {$\left[\mathrm{g} / \mathrm{cm}^{3}\right]$} & {$[\mathrm{g}]$} & Width & Height & Depth \\
\hline Formed Polystyrene & 0.03 & 38 & 9.9 & 5.9 & 20.8 \\
\hline Ordinary Concrete & 1.90 & 2403 & 9.8 & 6.2 & 20.8 \\
\hline Elemental Lead & 11.44 & 5720 & 10.0 & 5.0 & 10.0 \\
\hline
\end{tabular}

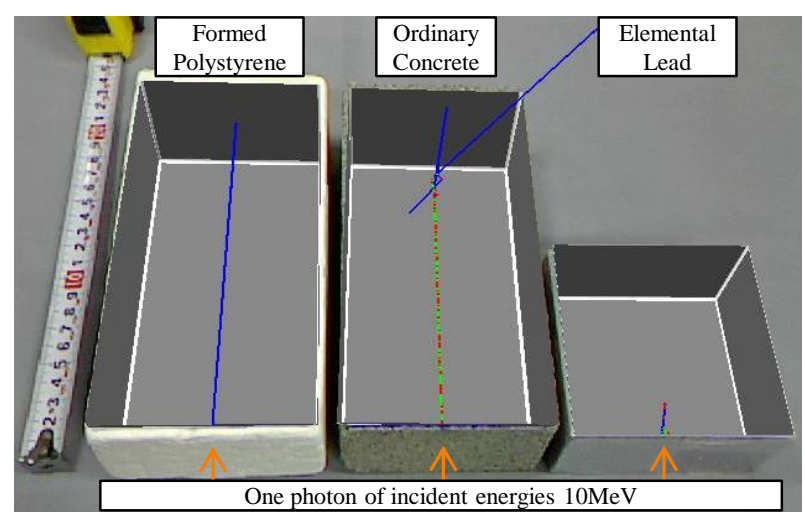

Figure 4. Tags of three kinds of shielding material with different density are recognized at the same time. Children can see the each development of cascades is different.

\section{Questionnaire surveys}

A public science event was held in 2011 at Ehime University which also included other organizations as is shown in Figure 6-(A). Children contemplated with interest. The number in attendance totaled 5,000 over the two days. Teaching assistants of our college explained about the basic properties of radiation for about 10 minutes and then demonstrated how to use the system. Many children had a chance to compare cascades for differing materials or differing incident energies of photons.

\subsection{Questionnaires after a public science event}

We had some questionnaires for the children in the public science event. By way of a survey, it appeared that it was the first time to see electromagnetic cascades for about the $90 \%$ of the participants and that it was very easy for them to understand how cascades change with differences in both density of shielding materials and incident energies. We also asked them to guess the length of the concrete block and the mass of the lead block. Figure 7 shows the results of the questionnaire. Figure 7 -(A) shows that $44.6 \%$ of the children answered the correct length within an error of $2 \mathrm{~cm}$. On the other hand, the rate of correct answers about the mass within an error of $1 \mathrm{~kg}$ was only $14.5 \%$, as shown in Figure 7-(B). This tends to indicate that most of the children who participated had difficulty associating the perceived mass with its numeric equivalent. This fact means this system is effective as educational tool to make understand the relationship between density and the radiation shielding ability for children.

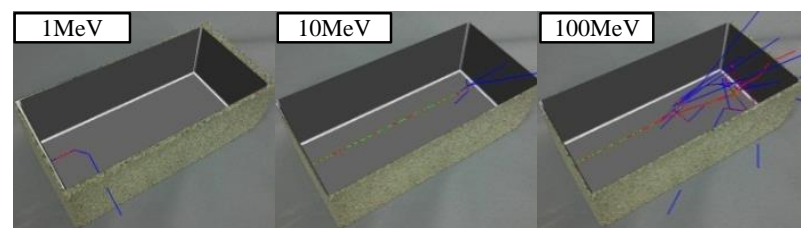

Figure 5. Children can observe different electromagnetic cascades with different incident energy by changing AR tag.
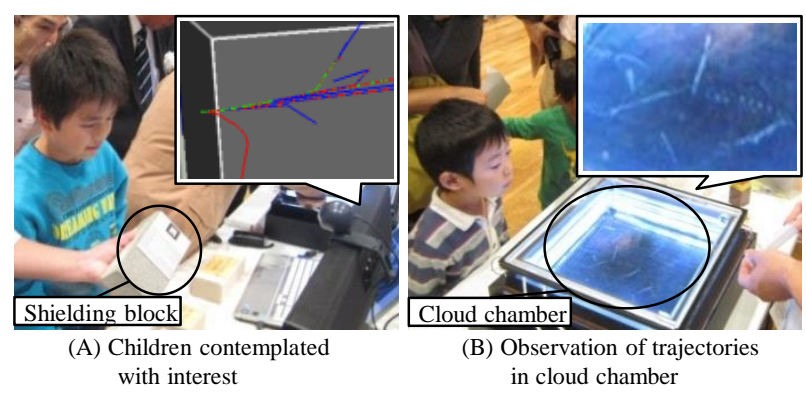

Figure 6. A public science event in 2011. Children can immediately understand the 3-D lines in the AR system are the trajectories of radiation particles after observation of trajectories in the cloud chamber.

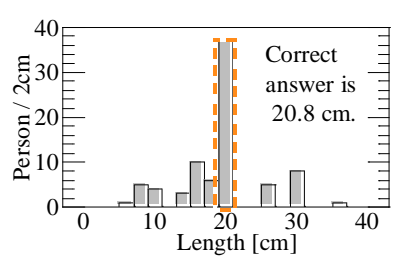

(A)Length of concrete block

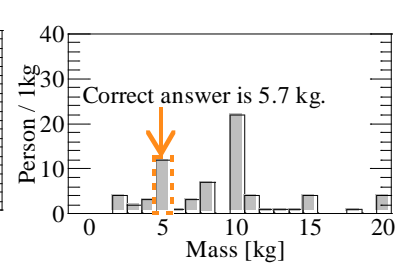

(B) Mass of lead block
Figure 7. Results of questionnaire for size and the mass of block. Children have difficulty associating a perceived mass.

\subsection{Questionnaires for the teaching assistants}

Some questionnaires were also prepared for teaching assistants. Some teaching assistants answered that it was difficult to make some children understand the 
decreasing kinematic energy of particles through cascades. Because the 3-D lines of trajectories in the composite image give a misconception for the children that the number of particles generated through cascades increase without a decrease in energy through the cascade process in the case of concrete or lead block. Besides, other teaching assistants answered some children can't imagine how electromagnetic cascades grow. 3-D lines pass through the both opposite surface of the block, especially in the case of polystyrene block in Figure 4. The result of the questionnaires for the teaching assistants revealed that the expression of 3-D lines may give a misconception about the energy of particles and incident direction of a photon for some children.

\section{Improvement as an educational tool}

\subsection{How to show the energy of particles}

According to our survey of the children who participated at our booth during a public science event in 2011, there are two problems with this system. First, some children did not recognize the absorption process by interactions with the shielding materials. Thus, a new function to support the comprehension of energy changes that occur during each interaction, a degree of transparency for each particle trajectory in the 3-D virtual image was set in accordance with the decreasing energy of the particles. The previous system and the new with transparency are shown in Figure 8-(A) and (B). It can be seen that each blue photon line from (a) to (d) in Figure 8-(B) is fading away proportional to the kinematic energy in order (a) to (d). This function may help learners to gain correct understanding of energy changes inside of the shielding materials.

The second problem was that some children were confused about the incident direction of the photon. It seems important for children to observe radiation particles directly. Cloud chambers are good educational tools to see moving radiation particles. After the observation of trajectories in a cloud chamber, the children appeared to understand, with no supplemental explanation, the trajectories of radiation particles drawn as 3-D lines in the AR system. Figure 6-(B) shows children observing trajectories in a cloud chamber at the public science event in 2011. Besides that, a new function, time-evolution, was added to enhance the children's understanding about radiation behavior. Electromagnetic cascades through shielding block are expressed by an animation as is shown in Figure 9 Children can observe how cascades grow.

\subsection{How to determine the cut-off energy}

The frame rate is mainly determined by the number of 3D line objects. The value of cut-off energy should be set to limit the number of lines so that the system can maintain a frame rate of more than $15 \mathrm{fps}$. In this AR system, the position resolution on the $\mathrm{PC}$ monitor is assumed about $1 \mathrm{~mm}$ for $640 \times 480$ pixels. Thus, the cut-off energy is set as the energy when the transport distance of the moving particles is less than $1 \mathrm{~mm}$. The cut-off energy for a photon is determined to be $20 \mathrm{keV}$ calculated from the mean free path for the concrete block. On the other hand, the transport distance of an electron or a positron under $100 \mathrm{keV}$ is limited to within $100 \mu \mathrm{m}$. So it is reasonable to set the cut-off energy to $20 \mathrm{keV}$ for both electrons and positrons.

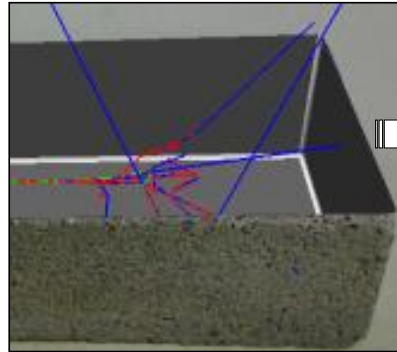

(A) Previous mode

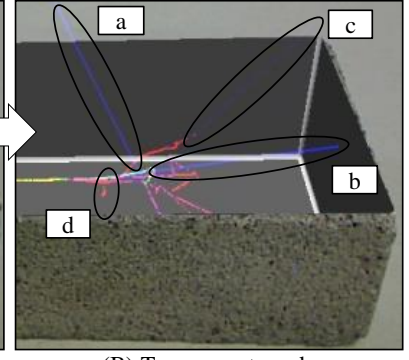

(B) Transparent mode
Figure 8. Transparent function supports the comprehension of energy changes that occur during each interaction.

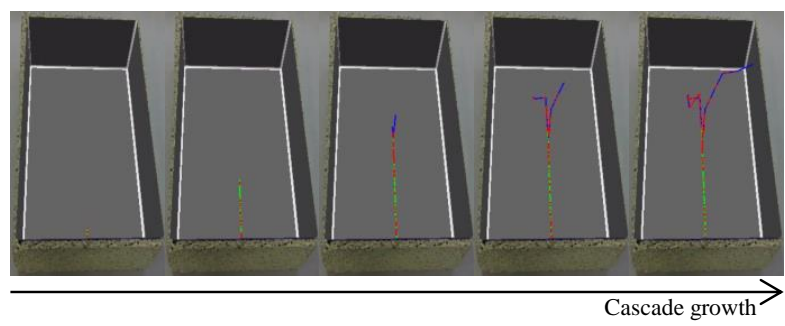

Figure 9. Children can observe how cascades grow by the time evolution function.

\section{Conclusion}

The tangible AR system was demonstrated to be suitable and effective for primary school educator use in promoting radiation awareness. According to the child survey, data indicated that the system seemed well suited for expressing and conveying the energy of the particles and growth of the cascades. The incident photon was assumed to be collimated in this system. In order to increase the sense of irradiation from a radiation source while using this system, a set of virtual radiation sources and a real collimator can be developed in the future as an additional component for this system. That type of system could be used as a tool to teach how to make a collimated beam with a real collimator.

\section{References}

[1] H. Kato and M. Billinghurst, Marker Tracking and HMD Calibration for a video-based Augmented Reality Conferencing System, IWAR 99, (1999).

[2] H. Hirayama and Y. Namito, Lecture Notes of EGS4 Course at KEK (Japanese Part), KEK Internal 99-5, (1999).

[3] H. Hirayama and Y. Namito, Lecture Note Education for Radiations using EGS4PICT32, KEK Internal 2001-11, (2011). 